\title{
Fogitare
}

\section{PERCEPÇÃO DOS PROFISSIONAIS DE ENFERMAGEM SOBRE A UTILIZAÇÃO DO CHECKLIST DO PARTO SEGURO}

\author{
Renan Joseph de Moraes Custódio ${ }^{1}$ (i) \\ Letícia Barbosa Kapassi² (1) \\ Danielly Teles Alves ${ }^{1}$ \\ Ângela Ferreira Barros ${ }^{1}$ (D) \\ Manuela Costa Melo ${ }^{1}$ (1) \\ Lara Mabelle Milfont Boeckmann ${ }^{3}$ \\ Luciana Melo de Moura ${ }^{4}$ (i)
}

\begin{abstract}
RESUMO
Objetivo: compreender como a implantação do Checklist do Parto Seguro modificou a prática obstétrica na percepção da equipe de enfermagem.

Método: estudo qualitativo, realizado entre outubro de 2018 e junho de 2019, com 36 profissionais de enfermagem de dois hospitais públicos de ensino no Distrito Federal - Brasil. Utilizou-se técnica narrativa e análise de conteúdo.

Resultados: os profissionais compreenderam que o instrumento trouxe benefício e contribuiu para maior qualidade e segurança na assistência obstétrica, além do estímulo à cultura de segurança. Relataram que a sua utilização provocou mudanças na rotina, as quais contribuiram para que alguns membros da equipe demonstrassem resistência e dificuldades pós-implantação.

Conclusão: as narrativas suscitaram reflexões como o planejamento em saúde, visando maior adesão da equipe de enfermagem às práticas seguras e sensibilização com relaçào à importância da ferramenta do Checklist do Parto Seguro.
\end{abstract}

DESCRITORES: Segurança do Paciente; Lista de Checagem; Serviços de Saúde Materno-Infantil; Parto Obstétrico; Enfermagem Obstétrica.

\section{PERCEPTION OF NURSING PROFESSIONALS ON THE USE OF THE SAFE DELIVERY CHECKLIST}

\section{RESUMEN:}

Objetivo: comprender cómo la implementación de una Lista de Verificación del Parto Seguro modificó la práctica obstétrica en la percepción del equipo de enfermería. Método: estudio cualitativo, realizado entre octubre de 2018 y junio de 2019, con 36 profesionales de enfermería de dos hospitales públicos de enseñanza del Distrito Federal - Brasil. Se utilizó la técnica narrativa y el análisis de contenido. Resultados: Los profesionales entendieron que el instrumento aportaba beneficios y contribuía a una mayor calidad y seguridad en la atención obstétrica, además de estimular la cultura de la seguridad. Informaron de que su uso provocó cambios en la rutina, lo que contribuyó a que algunos miembros del equipo mostraran resistencia y dificultades posteriores a la implantación. Conclusión: las narrativas suscitan reflexiones como el planeamiento en salud, visando una mayor adhesión del equipo de enfermería a las prácticas seguras y la sensibilización con relación a la importancia de la herramienta de una lista de verificación del Parto Seguro.

DESCRIPTORES: Seguridad del Paciente; Lista de Verificación; Servicios de Salud Materno - Infantil; Parto Obstétrico; Enfermería Obstétrica. 
A assistência ao parto engloba os momentos pré, intra e pós-parto, que exigem práticas essenciais para garantir a segurança às usuárias dos serviços de saúde. Contudo, nos países em desenvolvimento, há demanda por melhorias na oferta e na qualidade da atenção obstétrica e neonatal, a fim de se reduzir as altas taxas de morbimortalidade ${ }^{(1-3)}$. Nesse contexto, o Brasil, por meio de suas políticas na área obstétrica, é signatário da Organização Mundial de Saúde (OMS) e participou do pacto mundial para o cumprimento dos objetivos do milênio, que entre outras metas, pretendia reduzir em $75 \%$ as taxas de mortalidade materna e neonatal até 2015 , entretanto, não obteve o êxito esperado(4).

Dentre as estratégias recomendadas pela OMS, uma das mais difundidas é o uso do Checklist do Parto Seguro, um instrumento simples e de baixo custo que pode ser adaptado às diversas realidades dos serviços de saúde em nível mundial. O uso do checklist contribui para a segurança no momento do parto, prevenção e/ou redução de incidentes e eventos adversos e, por conseguinte, diminuição no índice de mobimortalidade relacionado. Possui itens que contemplam atendimento seguro à parturiente e recém-nascido e relaciona-se ao manejo das principais causas dos óbitos maternos, tais como hemorragias, infecções, doenças hipertensivas e complicações maternas e neonatais relacionadas ao parto e pósparto ${ }^{(5-7)}$.

Mesmo com resultados positivos de adesão ao checklist, existem dificuldades no monitoramento da manutenção desta adesão com o passar do tempo. No Brasil, por exemplo, de 978 prontuários revisados, $71 \%$ possuíam Cheklists do Parto Seguro com preenchimento de $24 \%$ em média dos itens, contra $0,1 \%$ de preenchimento completo, 0 que sugeriu treinamento insuficiente dos profissionais ${ }^{(5)}$.

As evidências existentes sobre a temática não elucidam em sua totalidade as lacunas do conhecimento, especialmente no que se refere às percepções dos profissionais acerca do uso do Cheklist do Parto Seguro, o que pode interferir na adesão, justificando-se a realização deste estudo. Desse modo, o estudo objetivou compreender como a implantação do Cheklist do Parto Seguro modificou a prática obstétrica na percepção da equipe de enfermagem.

\section{MÉTODO}

Estudo descritivo exploratório com abordagem qualitativa, utilizando-se como referencial metodológico a técnica da narrativa ${ }^{(8)}$. Realizada em dois hospitais públicos de referência materno-infantil, nas unidades de centro obstétrico e de alojamento conjunto, no Distrito Federal, Brasil. Para manter o anonimato das instituições, foram denominados de hospital A e hospital B. Ambos possuíam implantação do Cheklist do Parto Seguro em suas unidades desde o final de 2017.

Nessas unidades de saúde, o fluxo de atendimento à parturiente começa no pronto socorro obstétrico. Após classificação de risco, são encaminhadas às salas de parto, onde as principais atividades são realizadas: coleta de exames e orientações, aplicação de estratégias para alívio não-farmacológico da dor, tais como bola, cavalinho, banqueta, chuveiro e massagem. O centro obstétrico do hospital A possui 85 técnicos de enfermagem e 16 enfermeiros assistênciais; no hospital B, são 45 técnicos e 11 enfermeiros.

Após o nascimento, a puérpera e o recém-nascido permanecem no centro obstétrico até que sua condição estabilize, sendo então encaminhados e acomodados no alojamento conjunto. $O$ alojamento conjunto do hospital A possui 40 técnicos de enfermagem e 12 
enfermeiros assistenciais; no hospital B, são 24 técnicos e oito enfermeiros.

A população foi selecionada por conveniência e para o recrutamento inicial, realizouse o contato com as chefias das respectivas unidades e envio do convite a todos da equipe de enfermagem nos três turnos de trabalho. A amostra envolveu 36 profissionais de enfermagem entre técnicos e enfermeiros, e foi determinada a partir da saturação das narrativas obtidas por ocasião das interpretações das categorias analíticas. Todos os participantes convidados para o estudo aceitaram compor a amostra, não havendo, portanto, recusas.

Os critérios de inclusão foram: profissionais com vínculo empregatício, atuantes há pelo menos seis meses no Centro Obstétrico e/ou Alojamento Conjunto, e que utilizavam em suas rotinas profissionais o Cheklist do Parto Seguro. Excluíram-se profissionais de enfermagem em férias, ou qualquer outro tipo de licença, seja médica, nojo, gala ou acompanhamento e enfermeiros residentes.

A coleta de dados foi realizada entre outubro de 2018 e junho de 2019, seis meses pós-implantação do checklist. Até a realização da coleta de dados deste estudo, o Núcleo de Qualidade e Segurança do Paciente havia promovido dez sessões de capacitação para os profissionais da assistência obstétrica com enfoque no preenchimento e adesão ao checklist.

Para a coleta de dados, elaborou-se um roteiro semi-estruturado, contendo informações sobre a caracterização dos participantes (idade, estado civil, grau de escolaridade, profissão e tempo de atuação no setor de trabalho) e a informação subjetiva acerca da percepção do profissional sobre a utilização do checklist: o que você acha da sua prática com a implantação do Cheklist do Parto Seguro? Cada entrevista durou, em média, 25 minutos. Previamente à coleta de dados, conduziu-se um estudo piloto para adaptação e readequação do intrumento.

A coleta, transcrição e codificação de dados foram conduzidas por um enfermeiro residente e dois estudantes de enfermagem de uma instituição pública de ensino superior do Distrito Federal, previamente capacitados pelos pesquisadores responsáveis pela orientação e coorientação do estudo. Esses pesquisadores os instruíram com base nos seguintes tópicos: Programa Nacional de Segurança do Paciente; como realizar a abordagem aos participantes e a entrevista; o Cheklist do Parto Seguro; e a relevância do diário de campo. Todos os dados coletados e transcritos foram conferidos por três pesquisadoras, que também foram responsáveis pela interpretação e estruturação da análise de conteúdo das narrativas.

As narrativas foram gravadas e trancristas na sua integralidade e os registros das impressões dos pesquisadores foram descritos no diário de campo, que contribuiu para análises mais aprofundadas. Realizaram-se leituras consecutivas, com recortes do conteúdo do texto e, ainda, a estruturação das informações colhidas. Para garantir o anonimato, os participantes foram designados com a letra $P$ e seus respectivos números que variaram de 1 a 36, seguidos de traços, e com identificação de pertencer ao hospital A ou B, por exemplo, P1-A.

As narrativas colhidas foram tratadas por meio da interpretação e estruturação da análise de conteúdo(9). Esse tipo de análise é dividido em três aspectos: pré-análise, exploração do material e tratamento das informações. A fase de pré-análise selecionou as informações, levando em consideração a relevância, a representatividade, a homogeneidade e a pertinência dos dados. A exploração do material consistiu na codificação dos resultados por meio de recorte, agregação ou enumeração dos textos. O terceiro aspecto é o momento de interpretação dos dados a partir da teoria estabelecida pelo pesquisador, a teoria da narrativa.

Para obter o rigor científico desejado para estudos qualitativos, utilizou-se o Standards for Reporting Qualitative Research (SRQR)(10). O projeto foi submetido ao Comitê de Ética e 
Pesquisa da Fundação de Ensino e Pesquisa do Distrito Federal e recebeu o protocolo de aprovação $n^{\circ} 2.885 .771 / 2018$ para a pesquisa realizada no hospital $A$, e os protocolos de aprovação de parecer $n^{\circ} 2.885 .743 / 2018$ e $n^{\circ} 3.137 .852 / 2019$ para o hospital B.

\section{RESULTADOS}

As 36 participantes são do sexo feminino, sendo 14 (38,9\%) enfermeiras, dessas oito $(57 \%)$ generalistas e seis $(43 \%)$ especialistas, e $22(61,1 \%)$ técnicas de enfermagem, com idade entre 25 e 65 anos. Com relação ao grau de escolaridade, 26 (72,2\%) tinham nível superior, dentre essas, nove $(25,0 \%)$ eram especialistas e uma $(2,8 \%)$ mestre. Todas servidoras públicas e atuavam no setor há mais de seis meses, sendo $20(55,6 \%)$ no hospital A e $16(44,4 \%)$ no hospital B.

Após análise interpretativa das narrativas, emergiram duas categorias analíticas: Percepção da equipe de enfermagem sobre a implantação do Checklist do Parto Seguro e Contribuições do Checklist do Parto Seguro para a assistência de enfermagem.

\section{Percepção da equipe de enfermagem sobre a implantação do Checklist do Parto Seguro}

Essa temática reuniu os aspectos referentes à implantação do Checklist do Parto Seguro nos setores, ou seja, a percepção das participantes a respeito da capacitação para seu uso. As narrativas inferiram que houve treinamentos para o uso do checklist, porém, com relatos de críticas ao modo como foi conduzido e também a quantidade restrita de capacitações. Apontaram que houve resistência e dificuldades com relação à adesão ao instrumento por parte de alguns membros, justificada pelo déficit de recursos humanos, demanda de trabalho elevada, falta de motivação e sensibilização para o uso do checklist, bem como a falta de envolvimento da equipe no processo de sua implantação. Constataramse também relatos de desmotivação por não haver liberação do trabalho para participar dos treinamentos e não compreensão da necessidade e finalidade do checklist. Foram identificadas como unidade de significância: treinamento, recursos humanos, adesão e dificuldades, conforme os seguintes achados:

Eu acho que poderia ter batido um pouco mais na tecla. Tem pessoas que não fazem da forma como tem que ser feito. Então acho que deveria existir mais treinamento. (P-12-B)

Aqui não consigo fazer todas as partes, temos muito serviço e testes rápidos para fazer. $(P 1-A)$

Eu preencho todos os passos sempre que possível, a rotina é muito puxada, o que faz a gente esquecer às vezes. (P8-A)

O checklist é o que eu te falo, é bom para você conhecer o paciente, mas fora isso eu acho que não tem mais nenhuma necessidade. (P6-B)

Eu participei das palestras, conheço os passos, mas ainda assim não vejo finalidade. (P14-A)

As dificuldades é a adesão que é baixa e a disponibilidade como em um plantão mais pesado que acaba sendo deixado de lado. (P7-B)

Mas o treinamento é desmotivador, pois não há liberação da escala de serviço e os profissionais não têm motivação para realizar. (P9-A)

A limitação é que nem sempre você tem aquele tempo suficiente para completá-lo. Talvez a crítica seja a implantação de repente, deveria ter mais treinamentos. (P13-B) 
Ficou a desejar, simplesmente jogaram pra gente, falou, agora vai ter o checklist, tá aqui, é em tal lugar que você vai aqui no computador, entendeu? Pra poder fazer o checklist. Mas ninguém sentou e conversou e explicou pra gente o porquê. Faltou envolver mais a equipe. (P15-B)

\section{Contribuições do Checklist de Parto Seguro para a assistência de enfermagem}

Nesta categoria, agruparam-se as narrativas sobre as contribuições do instrumento Checklist do Parto Seguro para a assistência de enfermagem. Os profissionais relataram benefícios oriundos do uso do checklist para nortear a assistência: perceberam maior atenção na identificação de falhas e fragilidades no cuidado à parturiente; vislumbraram no checklist a possibilidade de evitar esquecimentos; referiram importância para as fases do checklist, um direcionamento padronizado na assistência, maior interação e melhor comunicação entre os integrantes da equipe. Emergiu das narrativas que tais benefícios contribuem para uma assistência de enfermagem qualificada. As unidades de significância foram: comunicação, atenção, padronização, sequência, direcionamento e qualificação, conforme as seguintes narrativas:

Participei do curso de segurança do paciente, foi muito bom, mostrou algumas falhas no nosso cuidado. (P8-A)

O checklist faz com que você preste mais atenção no que tá sendo feito, pra não ter esquecimento. (P1-A)

Eu acho que as quatro partes do checklist são importantes, são um pouco repetitivas, mas importantes. (P2-A)

Eu gosto do instrumento, porque você checa aquilo que já foi feito, na sequência, faço o meu pra colega subsequente ver o que tá acontecendo, entendeu? (P5-B)

Trouxe benefícios, lógico, muito. Por causa desses pontos, que ficava falho algumas coisa. Muito paciente pra pouco funcionário. Agora com o padronizado ficou mais fácil. (P5-B)

Direciona uma assistência mais qualificada. Porque quando a paciente interna, a gente já observa se ela faz uso de algum antibiótico, se ela tem algum problema de saúde tipo pressão alta, diabetes. (P10-B)

Então eu acho que esse modelo de checklist ele facilita a comunicação, porque ele é fechado e qualquer pessoa que lê entende. (P13-B)

Há uma interação maior entre a equipe. (P17-B)

Este estudo permitiu compreender a percepção da equipe de enfermagem sobre a sua prática com a implantação do Checklist do Parto Seguro nos cenários estudados e pôde contribuir para que os gestores se familiarizem com os fatores que têm influenciado a adesão por parte da equipe de enfermagem e oportunizar o desenvolvimento de atividades a fim de atenuar/corrigir os fatores que a dificultam. Faz-se necessário reforçar que o checklist é parte da sistematização do cuidado de enfermagem, que possibilita diminuição dos eventos adversos no cuidado prestado.

As narrativas analisadas a partir da primeira categoria temática demonstraram que a inserção de uma nova tecnologia ou ferramenta por si só não garante que o seu uso será efetivado. O envolvimento das pessoas no processo é fundamental para reconhecer aquela 
prática como importante, caso contrário, a adesão ao preenchimento pode ser baixa e não transparecer a necessidade a que se propõe $e^{(5,11-12)}$, o que se desvelou nas narrativas destes profissionais representadas pelo apelo às dificuldades frente ao uso do checklist.

Apesar das capacitações, existiram profissionais que não perceberam o propósito do instrumento e referiram desmotivação para os treinamentos e baixa adesão relacionada à sobrecarga de trabalho. Verificou-se que a baixa adesão ao checklist foi oriunda da desmotivação relacionada ao acréscimo de novas práticas à sua rotina diária que, em suas visões, contribuíram para aumentar a carga de trabalho. Essa interpretação negativa acerca do checklist, como sendo desnecessário e/ou burocrático, também foi identificada em outro estudo e contribuiu para diminuir a adesão e eficácia da ferramenta ${ }^{(5)}$.

Para que o Checklist do Parto Seguro seja usado da forma correta e atinja seu objetivo, é necessário que as instituições de saúde adotem a cultura de segurança e que haja capacitação dos profissionais para utilizá-lo. É preciso que os profissionais de saúde vejam essa ferramenta não como mais um mero documento a ser preenchido, mas como uma estratégia que irá diminuir a ocorrência do erro e, consequentemente, melhorar a qualidade da assistência(13).

Como sugestão para melhor utilização do instrumento, faz-se necessário o envolvimento dos profissionais, desde a elaboração inicial do projeto, os possíveis ajustes para as adequações à realidade da prática local, capacitação constante de toda a equipe multidisciplinar, e supervisão contínua do processo de trabalho envolvido.

O uso de videocases para a sensibilização dos profissionais e realização das práticas obstétricas seguras na assistência ao parto tem-se mostrado como recurso metodológico na educação permanente. O vídeo se constitue em tecnologia educativa que amplia a capacidade de compartilhar conhecimentos e experiências com alto número de espectadores, podendo ser disseminado pela internet. Assim, a produção de videocases pode ser considerado uma alternativa para incentivar a implantação do Checklist do Parto Seguro nas instituições de saúde e apoiar a educação permanente das equipes ${ }^{(14)}$.

Os profissionais apontaram dificuldades que podem influenciar na baixa adesão e preenchimentos inadequados relacionados à sobrecarga de trabalho. Igualmente, a aplicação do Checklist do Parto Seguro em 19 países, envolvendo 134 profissionais e 39 equipes, revelou desafios na visão dos participantes que apontaram, entre outros motivos, a incapacidade de usar o checklist quando estavam muito ocupados e $30 \%$ relataram recursos humanos insuficientes para apoiar a sua implementação(7). Resultados similares foram encontrados em outro estudo realizado no Brasil, que demonstrou uma porcentagem de $4 \%$ de preenchimento completo do checklist, presente em $61,4 \%$ dos prontuários das cirurgias avaliadas ${ }^{(15)}$.

Outro resultado semelhante foi encontrado no Sri Lanka, Ásia, que evidenciou os relatos de aumento da carga de trabalho e o pouco entusiasmo dos profissionais de saúde em relação aos novos acréscimos na rotina pelo uso do Checklist do Parto Seguro. Constatou-se que a atitude em relação ao instrumento foi satisfatória. No entanto, a taxa de adoção entre todos os trabalhadores foi de $45,8 \%$ e o conhecimento sobre o checklist foi de $60,1 \%$. Portanto, apontou-se como necessário aumentar a conscientização sobre o valor e o uso correto da ferrramenta, atentando para os níveis adequados de recursos humanos ${ }^{(16)}$.

$\mathrm{Na}$ Índia, um estudo randomizado controlado com treinamento de profissionais objetivou melhorar a adoção e o uso sustentado do Checklist do Parto Seguro, e demonstrou que apenas o instrumento não é capaz de melhorar a assistência, mas depende da utilização correta e motivacional. Foi dada ênfase na construção de relacionamentos e respeito mútuo, o que levou à confiança entre os treinadores e os profissionais, ajudando a influenciar mudanças ${ }^{(17)}$.

Identificando os motivos da não adesão ou a causa da resistência, é possível facilitar o 
uso de estratégias que se voltem para as atividades que requerem um contínuo treinamento dos profissionais para sensibilização, adaptação e manutenção do seu uso no cuidado(5). Isso ressalta a relevância dos estudos qualitativos que identificam as percepções dos que utilizam o Checklist do Parto Seguro em suas rotinas profissionais. Cabe destacar um estudo, no qual o processo de implementação com o suporte de atividades de sensibilização com apoio da chefia do setor, treinamentos e feedbacks constantes proporcionaram maior adesão ao checklist(6).

Referente à segunda categoria temática (Percepção dos profissionais sobre as contribuições do Checklist do Parto Seguro para a assistência de enfermagem), revelouse a atribuição de importância ao instrumento e a indicação de benefícios dele para o processo de trabalho. Similarmente, estudos indicaram que o uso do checklist melhora a comunicação e relação interprofissional e o direcionamento das ações, tendo em vista que reúne informações fundamentais de rápida e fácil aplicação ${ }^{(1,5,18)}$.

Dados semelhantes foram encontrados em estudo multicêntrico conduzido em 19 países, evidenciando que quase 3/4 dos entrevistados consideraram que o uso do Checklist do Parto Seguro potencializou a conscientização sobre a segurança do paciente, e mais de $2 / 3$ dos entrevistados identificaram que melhorou a comunicação e o trabalho em equipe ${ }^{(7)}$. Outra evidência apontou que em torno de $69,4 \%$ dos profissionais concordaram que o checklist estimulou a comunicação interpessoal e o trabalho em equipe ${ }^{(16)}$.

Estudo brasileiro apontou que o Checklist do Parto Seguro é um instrumento de fácil aplicação, que facilita a rotina de trabalho na perspectiva do profissional e contribui para evitar o esquecimento e agilizar a abordagem de uma grande quantidade de fatores relativos ao contexto do cliente ${ }^{(19)}$. Duas pesquisas também apontaram melhora na qualidade da assistência prestada e redução de incidentes ${ }^{(5,20)}$, corroborando com os achados deste estudo.

Observou-se que a assistência de enfermagem exige constantes registros dos procedimentos de rotina no processo de trabalho. Para os profissionais da enfermagem, a comunicação representa mais do que um meio de transferir informações e interagir, mas também uma forma de organizar o cuidado. E quando o instrumento não é preenchido de maneira adequada, dificulta a assistência e fragiliza o monitoramento da segurança da paciente.

A partir desses dados, infere-se que os treinamentos sobre o preenchimento do checklist precisam ser contínuos. Quando a equipe é envolvida, conscientizada e se sente motivada a adotar uma nova prática, é possível atingir alto grau de adesão mesmo que não seja instituída qualquer espécie de recompensa individual(1,21). Valorizar a educação em saúde é uma estratégia político-pedagógica que toma como objeto os problemas e necessidades emanados do processo de trabalho em saúde ${ }^{(22)}$. Diante disso, ressalta-se a relevância dos profissionais que executam as políticas públicas serem ouvidos e suas necessidades compreendidas por meio de feedbacks contínuos e comunicação assertiva nas relações profissionais.

As limitações deste estudo são próprias das investigações qualitativas, que incluem amostra restrita definida de maneira não probabilística e pela saturação das narrativas obtidas, impondo desafios à possibilidade da generalização dos resultados, além das características das análises interpretativas das subjetividades que envolvem os universos de pesquisadores e participantes. Ademais, participaram do estudo apenas profissionais da equipe de enfermagem, excluindo-se outras categorias profissionais. Não obstante, é preciso destacar que a equipe de enfermagem correspondeu à categoria com maior força de trabalho e majoritariamente responsabilizou-se pelo preenchimento do checklist. 
Este estudo cumpriu seu propósito ao compreender como a implantação do Checklist do Parto Seguro modificou a prática obstétrica na percepção da equipe de enfermagem e demonstrou achados que corroboraram com outras evidências. É relevante por trazer as narrativas dos profissionais, que reconheceram a importância desse checklist ao considerar que o instrumento trouxe benefícios e contribuiu para maior qualidade e segurança na assistência obstétrica, além do estímulo à cultura de segurança.

Tendo em vista que o instrumento demonstra eficácia para redução de incidentes e redução da mortalidade materna e neonatal, consonantes com a Política Nacional de Atenção Integral à Saúde da Mulher e da Criança, a implantação e monitorização desta ferramenta ultrapassam uma simples implantação e por isso urge promover capacitações, além do provimento apropriado de recursos humanos.

As implicações para futuros estudos e para a prática profissional tornam-se relevantes, haja visto escassas publicações verificadas na vertente qualitativa. Os resultados poderão contribuir para que gestores e profissionais de saúde compreendam os aspectos subjetivos que impactam e influenciam na operacionalização das políticas públicas, em especial, no tocante à adesão completa e adequada ao Cheklist do Parto Seguro. Os achados analisados à luz da pesquisa qualitativa agregam valores e auxiliam os gestores na formulação do planejamento em saúde ao instituir estratégias que aproximem a gestão às demandas dos trabalhadores.

Destaca-se que o Cheklist do Parto Seguro é parte da sistematização do cuidado de enfermagem em obstetrícia, e possibilita padronizar uma assistência segura e de qualidade pautada nas melhores evidências científicas. Tais achados não podem ser ignorados, pois poderão auxiliar outros serviços a organizarem uma implantação do checklist considerando as necessidades dos trabalhadores que o operacionalizam.

\section{REFERÊNCIAS}

1. Pereira RM, Fonseca G de O, Pereira ACCC, Gonçalves GA, Mafra RA. Novas práticas de atenção ao parto e os desafios para a humanização da assistência nas regiões sul e sudeste do Brasil. Ciênc. saúde coletiva. [Internet]. 2018 [acesso em 06 mar 2019]; 23(11). Disponível em: https://doi.org/10.1590/1413812320182311.07832016.

2. Batista Filho M, Rissin A. WHO and the epidemic of cesarians. Rev Bras Saude Mater Infant. [Internet]. 2018 [acesso em 06 mar 2019]; 18(1). Disponível em: https://doi.org/10.1590/1806-93042018000100001.

3. Bétran AP, Temmerman M, Kingdon C, Mohiddin A, Opiyo N, Torloni MR, et al. Interventions to reduce unnecessary caesarean sections in healthy women and babies. Lancet. [Internet]. 2018 [acesso em 06 mar 2019]; 392(10155). Disponível em: http://doi.org/10.1016/S0140-6736(18)31927-5.

4. Roma JC. Os objetivos de desenvolvimento do milênio e sua transição para os objetivos de desenvolvimento sustentável. Cienc Cult. [Internet]. 2019 [acesso em 21 jan 2020]; 71(1). Disponível em: http://dx.doi.org/10.21800/2317-66602019000100011.

5. Praxedes A de O, Arrais L, Araújo MAA de, Silva EMM da, Gama ZA da S, Freitas MR de. Avaliação da adesão à lista de verificação de segurança no parto em uma maternidade pública no Nordeste do Brasil. Cad Saúde Pública [Internet]. 2017 [acesso em 06 mar 2019]; 33(10). Disponível em: http://dx.doi. org/10.1590/0102-311X00034516.

6. Kabongo L, Gass J, Kivondo B, Kara N, Semrau K, Hirschhorn LR. Implementing the WHO Safe Childbirth Checklist: lessons learnt on a quality improvement initiative to improve mother and newborn care at Gobabis District Hospital, Namibia. BMJ Open Qual. [Internet]. 2017 [acesso em 06 mar 2019]; 6(2). Disponível em: http://dx.doi.org/10.1136/bmjoq-2017-000145. 
7. Muhorakeye F, Magriples U, Rulisa S, Ntasumbumuyange D, Bazzett-Matabele L. Impact of WHO Safe Childbirth Checklist on knowledge and practice of OB providers in Rwanda. Am J Obstet Gynecol. [Internet]. 2019 [acesso em 21 jan 2020]; 220(supl.1). Disponível em: https://doi.org/10.1016/j. ajog.2018.11.277.

8. Muylaert CJ, Sarubbi Júnior V, Gallo PR, Rolim Neto ML, Reis AOA. Narrative interviews: an important resource in qualitative research. Rev Esc Enferm USP. [Internet]. 2014 [acesso em 06 mar 2019]; 48(spe2). Disponível em: https://doi.org/10.1590/S0080-623420140000800027.

9. Bardin L. Análise de conteúdo. Lisboa: Edições 70; 2018.

10. O'Brien BC, Harris IB, Beckman TJ, Reed DA, Cook DA. Standards for Reporting Qualitative Research: A Synthesis of Recommendations. Academic Med. [Internet]. 2014 [acesso em 06 mar 2019]; 89(9).

Disponível em: http://dx.doi.org/10.1097/ACM.0000000000000388.

11. Ribeiro HCTC, Quites HF de O, Bredes AC, Sousa KA da S, Alves M. Adesão ao preenchimento do checklist de segurança cirúrgica. Cad. Saúde Pública [Internet]. 2017 [acesso em 28 jan 2021]; 33(10). Disponível em: https://doi.org/10.1590/0102-311x00046216.

12. Garcia T de F, Oliveira AC. Self-reported index of the orthopedic surgery team on the surgical safety checklist and implementation protocol. Cogitare enferm [Internet]. 2018 [acesso em 28 jan 2021]; 23(1). Disponível em: http://dx.doi.org/10.5380/ce.v23i1.52013.

13. Ferreira NCS, Ribeiro L, Mendonça ET, Amaro MOF. Checklist de cirurgia segura: conhecimento e utilização do instrumento na perspectiva dos técnicos de enfermagem. RECOM. [Internet]. 2019 [acesso em 31 jan 2021]; 9(e2608). Disponível em: http://dx.doi.org/10.19175/recom.v9i0.2608.

14. Costa ARC da, Imoto AM, Gottems LBD. Videocase sobre a lista de verificação do parto seguro: sensibilização dos profissionais da saúde. Enferm. Foco [Internet]. 2019 [acesso em 31 jan 2021]; 10(5). Disponível em: http://revista.cofen.gov.br/index.php/enfermagem/article/view/2355/627.

15. Freitas MR de, Antunes AG, Lopes BNA, Fernandes F da C, Monte L de C, Gama ZA da S. Avaliação da adesão ao checklist de cirurgia segura da OMS em cirurgias urológicas e ginecológicas, em dois hospitais de ensino de Natal, Rio Grande do Norte, Brasil. Cad. Saude Pública [Internet]. 2014 [acesso em 06 mar 2019]; 30(1). Disponível em: https://doi.org/10.1590/0102-311X00184612.

16. Patabendige $\mathrm{M}$, Senanayake $\mathrm{H}$. Implementation of the $\mathrm{WHO}$ safe childbirth checklist program at a tertiary care setting in Sri Lanka: a developing country experience. BMC Pregnancy Childbirth [Internet]. 2015 [acesso em 06 mar 2019]; 15(12). Disponível em: http://dx.doi.org/10.1186/s12884-015-0436-0.

17. Kara N, Firestone R, Kalita T, Gawande AA, Kumar V, Kodkany B, et al. The betterbirth program: pursuing effective adoption and sustained use of the WHO safe childbirth checklist through coachingbased implementation in Uttar Pradesh, India. Glob Heal Sci Pract. [Internet]. 2017 [acesso em 06 mar 2019]; 5(2). Disponível em: https://www.ncbi.nlm.nih.gov/pmc/articles/PMC5487086/.

18. Boeckmann LMM, Rodrigues MCS. Adaptation and validation of a surgical safety checklist in the cesarean delivery. Texto contexto-enferm. [Internet]. 2018 [acesso em 06 mar 2019]; 27(3). Disponível em: https://doi.org/10.1590/0104-070720180002780017.

19. Carvalho ICB de M, Rosendo TMS de S, Freitas MR de, Silva EMM da, Medeiros WR, Moutinho NF, et al. Adaptation and validation of the World Health Organization's Safe Childbirth Checklist for the Brazilian context. Rev Bras.Saúde Mater. Infant. [Internet]. 2018 [acesso em 06 mar 2019]; 18(2). Disponível em: http://dx.doi.org/10.1590/1806-93042018000200009.

20. Kumar S, Yadav V, Balasubramaniam S, Jain Y, Joshi CS, Saran K, et al. Effectiveness of the WHO SCC on improving adherence to essential practices during childbirth, in resource constrained settings. BMC Pregnancy and Childbirth [Internet]. 2016 [acesso em 06 mar 2019]; 16(345). Disponível em: http://dx.doi. org/10.1186/s12884-016-1139-x.

21. Campos KFC, Sena RR de, Silva KL. Permanent professional education in healthcare services. Esc Anna 
Nery [Internet]. 2017 [acesso em 06 mar 2019]; 21(4). Disponível em: http://dx.doi.org/10.1590/21779465-EAN-2016-0317.

22. Borges FA, Fortuna CM, Feliciano AB, Ogata MN, Kasper M, Silva MV da. Analysis of professional implication as a tool of permanent education in health. Rev Latino-Am Enfermagem [Internet]. 2019 [acesso em 21 jan 2020]; 27. Disponível em: http://dx.doi.org/10.1590/1518-8345.3114.3189.

\title{
COMO REFERENCIAR ESTE ARTIGO:
}

Custódio RJ de M, Kapassi LB, Alves DT, Barros AF, Melo MC, Boeckman LMM, et al. Percepção dos profissionais de enfermagem sobre a utilização do checklist do parto seguro. Cogitare enferm. [Internet]. 2021 [acesso em "colocar data de acesso, dia, mês abreviado e ano"]; 26. Disponível em: http://dx.doi.org/10.5380/ce.v26i0.74752.

Recebido em: 30/06/2020

Aprovado em: 02/02/2021

Editora associada: Tatiane Herreira Trigueiro

\author{
Autor Correspondente: \\ Manuela Costa Melo \\ Escola Superior em Ciências da Saúde - Brasília, DF, Brasil \\ E-mail: melomanuela91@gmail.com
}

Contribuição dos autores:

Contribuições substanciais para a concepção ou desenho do estudo; ou a aquisição, análise ou interpretação de dados do estudo - RJMC, LBK, DTA, AFB, MCM

Elaboração e revisão crítica do conteúdo intelectual do estudo - RJMC, LBK, DTA, AFB, MCM, LMMB, LMM Aprovação da versão final do estudo a ser publicado - RJMC, LBK, DTA, AFB, MCM, LMMB, LMM

Responsável por todos os aspectos do estudo, assegurando as questões de precisão ou integridade de qualquer parte do estudo - RJMC, LBK, DTA, AFB, MCM, LMMB, LMM

Copyright (C) 2021 Este é um artigo em acesso aberto distribuído nos termos da Licença Creative Commons Atribuição, que permite o uso irrestrito, a distribuição e reprodução em qualquer meio desde que o artigo original seja devidamente citado. 